$\xi=$ 离

\title{
Emphysematous gastritis developing after isolated cardioembolic event
}

\author{
Paul Muna-Aguon MD*, Tyler Aasen DO, Brenda Shinar MD \\ Banner University Medical Center, 1111 E McDowell Road, Phoenix, AZ 85006 \\ *Corresponding author E-mail: paulaguon@gmail.com
}

\begin{abstract}
Emphysematous gastritis is a very rare disease associated with a high mortality rate, making prompt recognition and early treatment of the underlying cause essential. We present a case of emphysematous gastritis developing after a cardio embolic-related acute ischemic injury to the gastric mucosa.
\end{abstract}

Keywords: Cardioembolic; Emphysematous gastritis; Gastric mucosa; Ischemic injury; Stomach

\section{Introduction}

Emphysematous gastritis (EG) is a rare phenomenon that is defined by gas within the gastric wall (Takano et al. 2015). EG is often a fatal condition caused by gas-producing bacteria with mortality rates as high as 55-61\% (Takano et al. 2015, Chen 2016, Watson et al. 2016). EG represents an extremely rare clinical finding, with approximately 80 cases reported in English literature. We present a case of emphysematous gastritis developing after a cardioembolic-related acute ischemic injury to the gastric mucosa.

\section{Case report}

A 48-year-old male with past medical history of end-stage renal disease (ESRD), diabetes, and atrial fibrillation presented with non-radiating epigastric pain that occurred during hemodialysis. He experienced two similar episodes within the previous 3 months. The pain was associated with abdominal distention, nausea, and one episode of blood-tinged emesis. He reported not taking his Coumadin for a couple of days prior to admission. Review of systems was negative for fevers, chills weight loss, hematochezia, melena, liver disease, caustic substance ingestion, diarrhea or constipation.

Physical Exam revealed normal vital signs, abdominal distention, and tenderness to palpation over the epigastric and LUQ regions without rebound or guarding. Laboratory investigation revealed white blood cell count (WBC) $14.4 \mathrm{~K} / \mathrm{mm} 3$, hemoglobin $(\mathrm{Hgb})$ $10.5 \mathrm{~g} / \mathrm{dL}$, platelets (Plt) $112 \mathrm{~K} / \mathrm{mm} 3$, prothrombin time (PT) 21.8 seconds with INR 1.7 , and lactic acid $0.7 \mathrm{mmol} / \mathrm{L}$. Liver function panel was unremarkable, renal function consistent with ESRD, and remaining complete metabolic panel (CMP) normal. Computed tomography (CT) of the abdomen and pelvis showed gas in the stomach wall and within the gastric veins consistent with emphysematous gastritis (Fig. 1 and Fig. 2).

The patient was admitted to medicine, placed on bowel rest, and started on intravenous Piperacillin-Tazobactam for empiric cover- age. He then underwent esophagogastroduodenoscopy (EGD) which showed erythematous mucosa in the cardia, gastric fundus, gastric body, greater curvature, and pylorus with clear demarcation concerning for ischemic injury. Biopsies were taken, which revealed superficial foveolar epithelium with regenerative changes, and cultures failed to grow organisms. Transthoracic echocardiogram showed no evidence of a thrombus or vegetation. Due to the ischemic appearance of the injury, a mesenteric angiogram was performed that revealed normal vasculature. The patient improved with conservative management and was restarted on Coumadin.

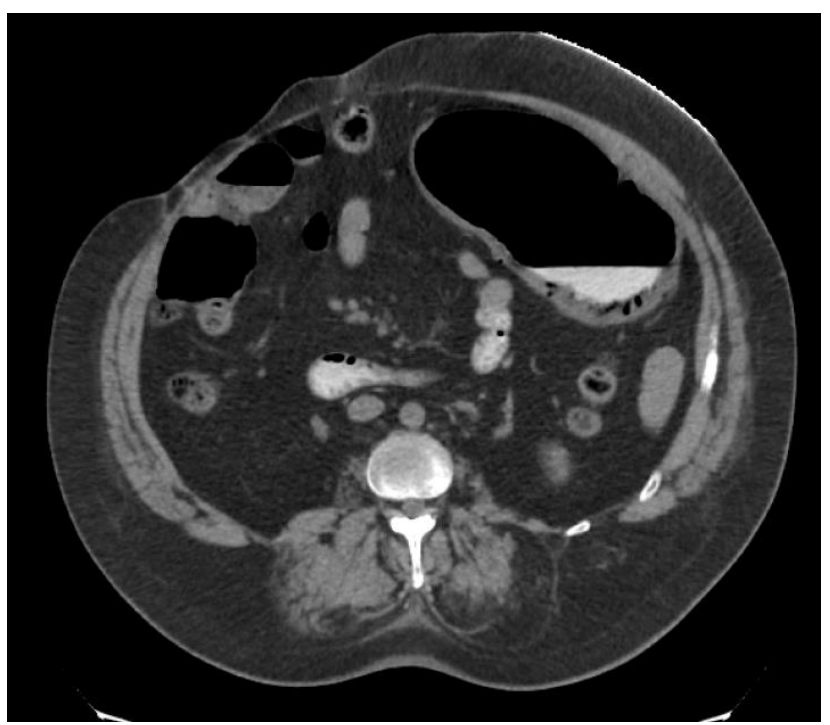

Fig. 1: Axial CT Scan Reveals Gas within the Wall of the Stomach. 


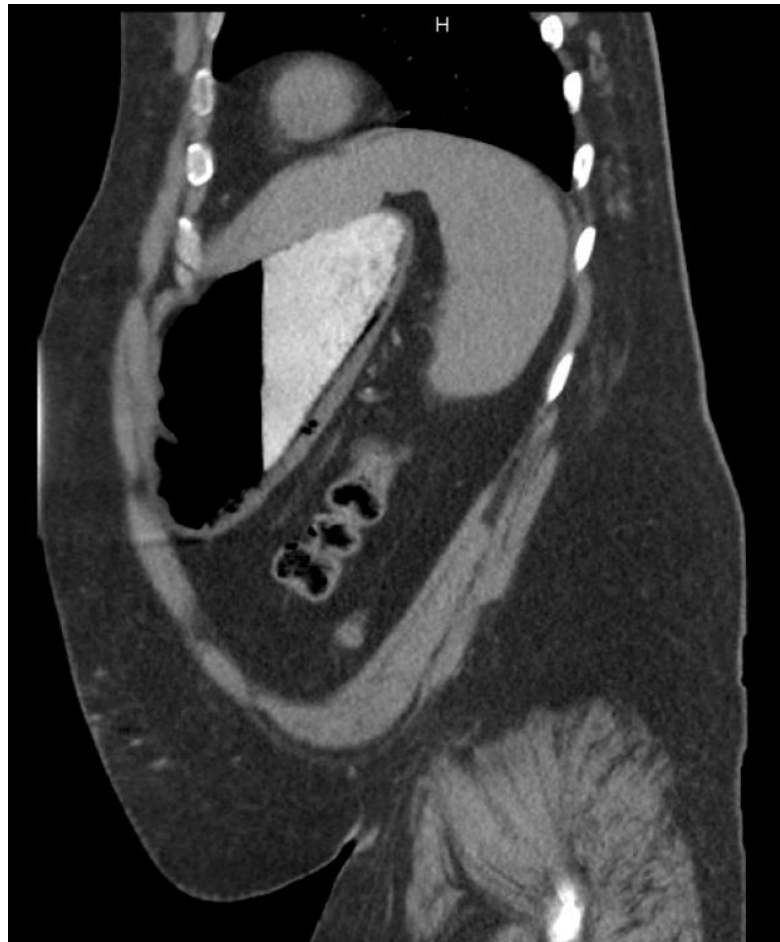

Fig. 2: Sagittal CT Scan Reveals Gas within the Wall of the Stomach.

\section{Discussion}

Emphysematous gastritis is a rare inflammatory disease caused by gas-producing organisms that was first described in 1889 by Frankel (Takano et al. 2015, Chen 2016, Watson et al. 2016) Though limited literature exists in the condition, some have described association of EG with ingestion of corrosives, alcoho abuse, diabetes, renal failure, gastroenteritis, recent abdominal surgery, long-term steroid use, pancreatitis, NSAID use, and gastric infarction (Guillen-Morales et al. 2015, Takano et al. 2015, Chen 2016, Watson et al. 2016). Bacteria are thought to enter the damaged gastric wall through mucosal ulcerations or hematogenous spread, resulting in secondary infection (Watson et al. 2016). Symptoms usually include epigastric pain, nausea, vomiting, diarrhea, leukocytosis, melena, and hematemesis (GuillenMorales et al. 2015, Watson et al. 2016). Although there is no consensus on a diagnostic imaging modality, CT is thought to be most sensitive and specific at detecting gas within the gastric wall (Takano et al. 2015, Uysal et al. 2015, Watson et al. 2016). EGD findings usually reveal erythematous, erosive lesions (Watson et al. 2016). Prior treatment involved surgical intervention until recently. Now, there has been more of a trend towards conservative management with broad-spectrum antibiotics, bowel rest, and nutritional support. Surgery is reserved for cases complicated by perforation, intestinal necrosis, or peritonitis (Guillen-Morales et al. 2015, Takano et al. 2015, Chen 2016, Watson et al. 2016).

In our case, the initial mucosal damage was likely due to cardioembolic event in the setting of atrial fibrillation and anticoagulation non-compliance with a subtherapeutic INR. CT showed gas in the gastric wall, and EGD showed signs of ischemic injury. Patient was started on broad-spectrum antibiotics a couple of days prior to biopsy of gastric lesion and was likely the reason cultures were negative.

\section{References}

[1] Takano Y, Yamamura E, Gomi K, Tohata M, Endo T, Suzuki R, Hayashi M, et al. Successful conservative treatment of emphysematous gastritis. Intern Med, 2015; 54(2):195-198. https://doi.org/ 10.2169/internalmedicine.54.3337.

[2] Watson A, Vadim B, Staudacher J, Carroll R, Yazici C. The predictors of mortality and secular changes in management strategies in emphysematous gastritis. Clin Res Hepatol Gastroenterol, 2016. https://doi.org/10.1016/j.clinre.2016.02.011.

[3] Chen H. Gastrointestinal: Emphysematous gastritis. J Gastroenterol Hepatol, 2016; 31(1):9. https://doi.org/10.1111/jgh.13170.

[4] Guillen-Morales C, Jimenez-Miramon FJ, Carrascosa-Miron T, Jover-Navalon JM. Emphysematous gastritis associated with portal venous gas: Medical management to an infrequent acute abdominal pain. Rev Esp Enferm Dig, 2015; 107(7):455-456. https://doi.org/ 10.17235/reed.2015.3540/2014.

[5] Uysal F, Akbal E, Gunes F, Sacar S, Ozdemir H. An unusual and serious cause of gastritis: Emphysematous gastritis. Diagn Interv Imaging, 2015; 96(3):291-292. https://doi.org/10.1016/j.diii.2014. 11.025 . 